Cite this: Phys. Chem. Chem. Phys., 2012, 14, 1778-1783

\title{
Excited state characterization of a polymeric indigo $\dagger$
}

\author{
R. Rondão, ${ }^{a}$ J. Seixas de Melo, ${ }^{* a}$ F. A. Schaberle ${ }^{a}$ and G. Voss ${ }^{b}$ \\ Received 17th October 2011, Accepted 25th November 2011 \\ DOI: $10.1039 / \mathrm{c} 2 \mathrm{cp23266a}$
}

A comprehensive investigation of the solution photophysics of a 5,5'-methylene-bridged polymeric indigo, a statistical copolymer consisting of indigo and $N$-acetylindigo units, was performed in organic solvents at room temperature and further compared with indigo. A complete spectral and photophysical characterization based on photoacoustic calorimetry, steady-state and time-resolved fluorescence data was undertaken. A fluorescence quantum yield of 0.00037 and an intersystem crossing singlet-to-triplet quantum yield of 0.006 (close to the value for indigo) were obtained, leading to a value of 0.9936 for the $S_{1} \rightsquigarrow S_{0}$ internal conversion (IC) quantum yield. Spectral and photophysical characteristics similar to indigo were obtained with, however, a special signature: it (mainly) decays single exponentially (in contrast with indigo, found to decay bi-exponentially), with a decay time value of $40-50 \mathrm{ps}$ and an even more efficient $S_{1} \leadsto S_{0}$ IC deactivation channel, related to an efficient energy migration within an energetic ladder of the polymer chromophoric segments. The photochemistry of this polymer, namely the degradation under light excitation, was also investigated and the obtained photoreaction quantum yield $\left(\phi_{\mathrm{R}}\right)$ in DMF was found to be 0.003 , which is lower than the previously determined value for indigo in the same solvent $\left(\phi_{\mathrm{R}}=0.0078\right)$. The overall data indicate that although the polymer and indigo have a close finger-print, the former is more stable which is suggested to be due to the additional intramolecular energy transfer process (within different chromophoric units) found with the polymer.

\section{Introduction}

Indigo, with more than 5000 years of history, is one of the oldest organic dyes to be used as a blue colorant for painting and dyeing. ${ }^{1-3}$ The longevity of this dye has historical reasons and has been linked to its worldwide widespread distribution, with more than 700 Indigofera (natural provenance of the dye) species known, to cultural aspects (linking for example the Western and Muslim civilizations), medicinal uses (for example of its isomer indirubin $^{4}$ with several applications including leukaemia treatment ${ }^{5}$ ) and also to the genesis of the German (and consequently European) chemical industry, recognized by the 1905 Nobel Prize in Chemistry awarded to A. von Baeyer for the first proposed synthetic indigo. The longevity of indigo is chemically related to its high stability towards light (photostability) which has been associated to the high efficiency of the radiationless internal conversion channel. ${ }^{6-8}$ In this context the quantum yield of a photochemical reaction $\left(\phi_{\mathrm{R}}\right)$ is an additional important

${ }^{a}$ Department of Chemistry, University of Coimbra,

P3004-535 Coimbra,Portugal.E-mail: sseixas@ci.uc.pt; Fax: 00351239827703

${ }^{b}$ Department of Bioorganic Chemistry, University of Bayreuth, D-95440 Bayreuth, Germany

$\dagger$ Electronic supplementary information (ESI) available: Spectral and photophysical data for indigo and polymeric indigo in DMSO, DMF and dioxane, together with PAC experiments. See DOI: 10.1039/ c2cp23266a parameter since it provides a quantitative parameter for the stability of molecules exposed to light excitation., 9,10

Due to the low solubility of indigo in majority of organic solvents the idea of a polymer built of indigo has probably never been properly equated. Despite the pioneer works of G. Heller ${ }^{11}$ in 1903 who prepared a polymeric indigo but could not realize the polymeric nature of the dye, ${ }^{12}$ the first report of a detailed characterization of a polymer of indigo is dated from 1990 with the work of Tanaka et $a l .{ }^{13}$ who prepared a polymer with an indigo unit in the main skeleton, and found that the polymer was insoluble but possessed a fraction that was attracted to a permanent magnet therefore displaying magnetic properties. More recently Voss et al. ${ }^{12}$ prepared and characterized two similar statistical copolymers made of indigo and $\mathrm{N}$-acetylindigo units with defined structures in solution and in the solid state.

With the recent recognition of the potential relevance and importance of organic conjugated polymers for photovoltaic applications, including isoindigo based conjugated polymers, ${ }^{14,15}$ a polymer of indigo, if displaying similar properties to its monomeric units, is of relevance since it would potentially generate long lived and stable charged species (whose radiative deactivation is negligible), a requisition which is mandatory for this type of applications.

\section{Experimental}

The polymeric indigo was synthesized and characterized as elsewhere reported. ${ }^{12}$ The polymers are statistical copolymers 
consisting of indigo- and $N$-acetylindigo units ( $1: 1)$. According to the solid state ${ }^{15} \mathrm{~N}$ NMR spectrum and in fresh solutions $\left({ }^{1} \mathrm{H}\right.$ NMR spectra in DMSO) the indigo units have keto structures, and the $\mathrm{N}$-acetylindigo units consist of the tautomeric indoxyl/indolone units. Additionally and regarding their properties in the solid state, from the IR spectra higher frequency $\mathrm{N}-\mathrm{H}$ absorption of polymers, as compared to indigo, could be observed which indicates weaker intermolecular hydrogen bonds [polymer: $3350 \mathrm{~cm}^{-1}$, monomer: $3244 \mathrm{~cm}^{-1}$ ]. Moreover, the polymers display a broad and strong absorption at $3500-2000 \mathrm{~cm}^{-1}\left(\mathrm{H}_{2} \mathrm{O}\right.$ and/or $\left.\mathrm{OH}\right)$ and amide-CO absorption at $1685 \mathrm{~cm}^{-1}$, together with low frequency ring-CO bands at $1620 \mathrm{~cm}^{-1}$ which verify the predominance of polar structures. For more details see ref. 12.

Indigo was purchased from Aldrich and used as received. The solvents used, DMF and DMSO, were of spectroscopic or equivalent grade.

Absorption and fluorescence spectra were recorded on Shimadzu UV-2100 and Horiba-Jovin-Yvon Spex Fluorog 3-2.2. spectrophotometers, respectively. Fluorescence spectra were corrected for the wavelength response of the system. The fluorescence quantum yields of compounds were determined using indigo $\left(\phi_{\mathrm{F}}=0.0023\right.$ in DMF) as standard. ${ }^{16}$

The fluorescence decays of the compounds were obtained with picosecond time resolution using an equipment described elsewhere, ${ }^{17}$ and were analyzed using the method of modulating functions implemented by Striker et al. ${ }^{18}$ The experimental excitation pulse (FWHM = 21 ps) was measured using a LUDOX scattering solution in water. After deconvolution of the experimental signal, the time resolution of the apparatus is ca. 2 ps.

Time-resolved PAC measurements were performed in a homemade apparatus following the front-face irradiation design described elsewhere. ${ }^{19}$ The solutions were pumped through a $0.11 \mathrm{~mm}$ thick cell at a $1 \mathrm{~mL} \mathrm{~min}^{-1}$ flow with a Kloehn V6 Syringe pump, irradiated with a EKSPLA PL2143A Nd : YAG laser $(\mathrm{FWHM}=30 \mathrm{ps})$ and collected with a Tektronix DPO 7254 oscilloscope. The acoustic waves were detected with a 2.25 MHz Panametrics transducer (model A106S) and preamplified with a Panametrics ultrasonic amplifier (model 5676). Sample and reference solutions were matched to better than $1 \%$ absorbances at a wavelength of $532 \mathrm{~nm}$. Data were analyzed using a software developed by Schaberle et al. ${ }^{20}$

For the reaction quantum yields $\left(\phi_{\mathrm{R}}\right)$, the following procedure was used. Irradiation was carried out in the HoribaJovin-Yvon Spex Fluorog 3-2.2. spectrophotometer in which $3 \mathrm{~mL}$ of each solution was irradiated at $335 \mathrm{~nm}$, with slits $2 \mathrm{~mm}$ (bandwidth of $4.2 \mathrm{~nm}$ ) and continuous magnetic stirring. The intensity of the incident light $\left(I_{0}\right)$ at $335 \mathrm{~nm}$ was measured using potassium hexacyanocobaltate(III) $\left(\mathrm{K}_{3}\left[\mathrm{Co}\left(\mathrm{CN}_{6}\right)\right]\right)^{21}$ as actinometer. The $I_{0}$ value was calculated, with correction to the absorbed light, according to eqn (1):

$$
I_{0}=V_{\mathrm{sol}}(\Delta A / \Delta \varepsilon) / 1000 \phi_{\mathrm{R}} \Delta t
$$

where $V_{\text {sol }}$ is the volume of irradiated solution in $\mathrm{mL} ; \Delta A$ is the change in absorbance at the monitoring wavelength over the irradiation time period, $\Delta t ; \Delta \varepsilon$ is the difference between the molar absorption coefficients of the reagent and the product at the monitoring wavelength; and $\phi_{\mathrm{R}}$ is the quantum yield of reaction. $^{10}$

The quantum yield of reaction for indigo was calculated with eqn (1) rearranged as:

$$
\phi_{\mathrm{R}}=V_{\mathrm{sol}}(\Delta A / \Delta \varepsilon) / 1000 I_{\mathrm{Abs}} \Delta t
$$

where $I_{\mathrm{Abs}}$ is the light absorbed by the solution at the irradiation wavelength; $I_{\mathrm{Abs}}$ was made equal to $I_{0} \times\left(1-10^{- \text {Airrad }}\right)$ when $A<2$ and to $I_{0}$ when $\mathrm{A}>2 .^{10}$

For the polymeric indigo the actinometry was made comparing directly the variance of absorption of both compounds polymer and indigo, and corrected with the optical density values (absorbance) at the irradiation wavelength, using eqn (3)

$$
\phi_{\mathrm{R}}=\frac{\Delta \mathrm{OD}_{\text {polymer }}}{\Delta \mathrm{OD}_{\text {indigo }}} \frac{\mathrm{Abs}_{\text {indigo }}}{\mathrm{Abs}_{\text {polymer }}} \phi_{\mathrm{R}}^{\text {indigo }}
$$

where $\Delta \mathrm{OD}_{\text {polymer }}$ is the change in the optical density $(\Delta \mathrm{OD})$ of the polymer solution with the irradiation time, $\Delta \mathrm{OD}_{\text {indigo }}$ is the change in the optical density of the solution of indigo with the time of irradiation, $\mathrm{Abs}_{\text {indigo }}$ is the optical density value (absorption) of indigo at the wavelength of irradiation $(335 \mathrm{~nm})$, $\mathrm{Abs}_{\text {polymer }}$ is the optical density value of the polymer at the wavelength of irradiation $(335 \mathrm{~nm})$ and $\phi_{\mathrm{R}}^{\text {indigo }}$ is the reaction quantum yield of indigo.

\section{Results and discussion}

The structure of the polymer is depicted in Scheme 1, showing units of indigo (keto structure) and of tautomeric $\mathrm{N}$-acetylindigo (consisting of $\mathrm{N}$-acetyl-donor units and indolone acceptor units).

\subsection{Spectral and photophysical properties}

The absorption and fluorescence of the polymer, together with the fluorescence excitation spectra in dimethylsulfoxide (DMSO), are shown in Fig. 1. Overlap between the absorption and the excitation is observed thus attesting the purity (and potentially little polydispersity) of the polymer. An identical behaviour was observed in other solvents such as dimethylformamide (DMF) and dioxane.

It is worth noting that from $\mathrm{UV} / \mathrm{V}$ is and ${ }^{1} \mathrm{H} \mathrm{NMR}$ data it was observed that in concentrated DMSO solutions the polymer forms aggregates after several weeks. ${ }^{12}$ Aggregation is accomplished by tautomerisation of the unsubstituted indigo units. Both tautomerization and aggregation are seen to be

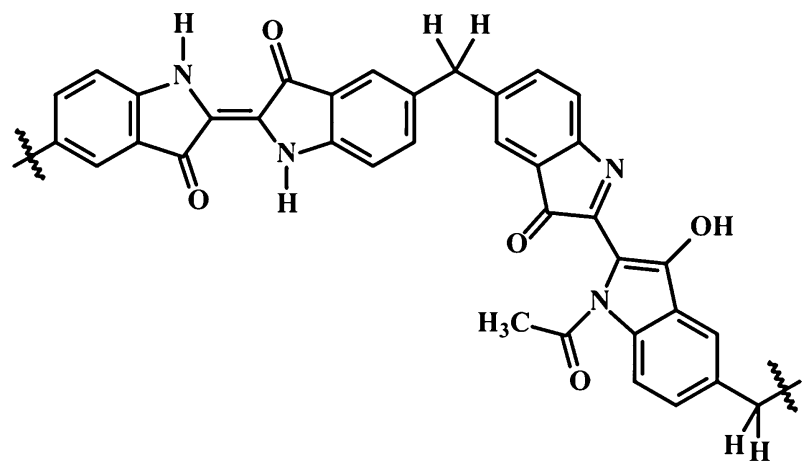

Scheme 1 


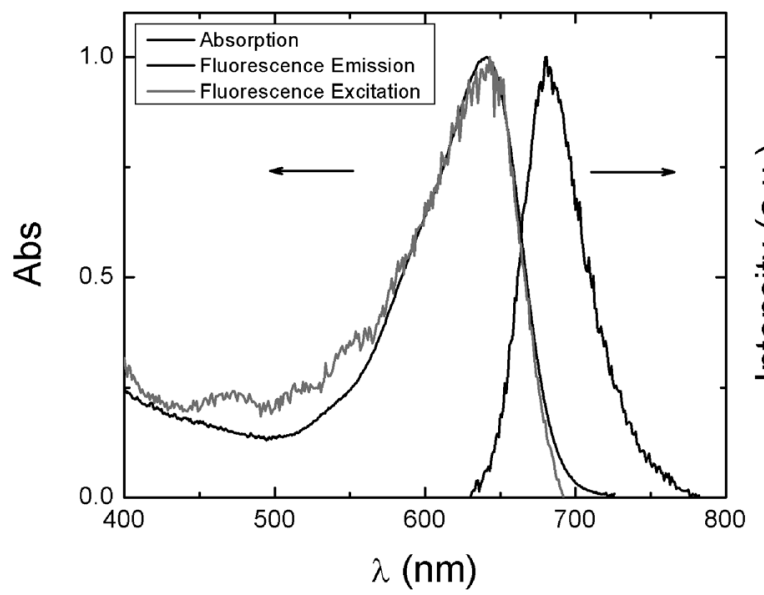

Fig. 1 Absorption and fluorescence emission and excitation spectra of the indigo polymer in DMSO, $T=293 \mathrm{~K}$.

reversible. ${ }^{12}$ However, it is also worth stressing that in the present work fresh solutions have been prepared and used and therefore only intramolecular interactions were investigated with no indication or observation of aggregation phenomena.

In Table 1 the spectral and photophysical data for the polymer and indigo itself are presented in DMF (see ESI $\dagger$ for other solvents). From data in Table 1 it can be seen that the absorption wavelength maximum is, for the polymer, redshifted $(20 \mathrm{~nm})$ relative to that observed for indigo (the value is higher in other solvents, see ESI $\dagger$ ). The same happens with the emission maximum, but now with a red-shift of $19 \mathrm{~nm}$ (again this value can be higher-up to $44 \mathrm{~nm}$ - depending on the solvent, see ESI $\dagger$ ). The absorption spectrum of the polymer shows, besides the red-shifted, a higher inhomogeneous broadening of its visible band, a situation which is common to observe in a polymer when compared to its oligomeric counterpart. ${ }^{22,23}$ Indeed, the well-defined conjugation length of an oligomer results in a smaller inhomogeneous broadening of the absorption spectra. ${ }^{24}$

The Stokes shift $\left(\Delta_{\mathrm{SS}}\right)$ is, with the polymer, comparable to that found with indigo, Table 1. However, whereas with indigo this has been linked to absorption being due to the keto neutral form, in the emission band this would be the result of the convoluted emission of this instantaneously formed species with that resulting from proton transfer (from the $\mathrm{N}-\mathrm{H}$ to the $\mathrm{C}=\mathrm{O}$ group which could be intra or - assisted by the solventintermolecular), in the case of the polymer the significant $\Delta_{\mathrm{SS}}$ value suggests to be the result of a different geometry in the ground and excited states. More interesting is the fact that in the emission (Fig. 2), the Full-Width Half-Maximum (FWHM) is smaller in the polymer than in indigo-albeit the fact that this difference is small — which shows that the emission of the

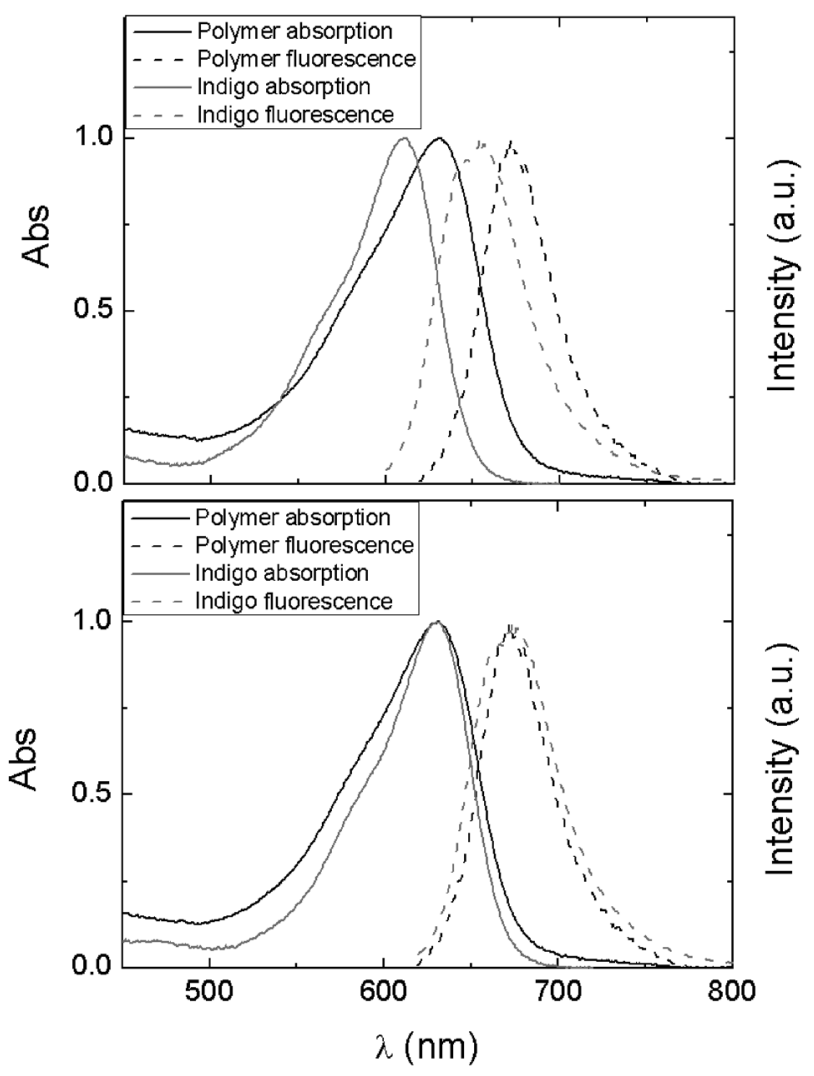

Fig. 2 Absorption and fluorescence spectra for indigo and the indigo polymer in DMF. In the top panel the spectra are normalized at its intensity/abs maxima, whereas in the bottom panel the absorption spectra of indigo and of its polymer are (artificially) set to have the same maxima and the same is valid for the emission spectra, where now the differences in the inhomogeneous broadening can be better visualized.

polymer is more localized. This decrease in the FWHM of the polymer suggests that now the emission is solely due to the emission of a single species (chromophoric units), in contrast to what is observed with indigo.

More interesting is the fact that the decays of the polymer are now basically single exponential (see Fig. 3) in contrast to what is observed with indigo where only with bi-exponential decay laws (with decay profiles being emission wavelength dependent) the decays could be properly fitted. ${ }^{16,25}$ Indeed, as can be seen in Fig. 4, the decays of indigo change when collected along the band, mirroring the same decay times but different pre-exponential factors. As mentioned above, this has been consistently linked, from various studies, to intra- or intermolecular proton transfer between the $\mathrm{N}-\mathrm{H}$ and $\mathrm{C}=\mathrm{O}$ groups of indigo. ${ }^{7,25,26}$ More recently from $a b$ initio electronic-structure

Table 1 Spectral and photophysical properties including quantum yields (fluorescence, $\phi_{\mathrm{F}}$, internal conversion, $\phi_{\mathrm{IC}}$, triplet formation, $\phi_{\mathrm{T}}$, and sensitized singlet oxygen formation, $\left.\phi_{\Delta}\right)$, lifetimes $\left(\tau_{\mathrm{F}}\right)$, rate constants $\left(k_{\mathrm{F}}, k_{\mathrm{NR}}, k_{\mathrm{IC}}, k_{\mathrm{ISC}}\right)$ for the polymeric indigo and indigo itself in DMF at $T=298 \mathrm{~K}$

\begin{tabular}{|c|c|c|c|c|c|c|c|c|c|c|c|c|c|}
\hline & $\lambda_{\mathrm{abs}} / \mathrm{nm}$ & $\varepsilon_{\mathrm{S}} / \mathrm{M}^{-1} \mathrm{~cm}^{-1}$ & $\lambda_{\mathrm{em}} / \mathrm{nm}$ & $\Delta_{\mathrm{SS}} / \mathrm{nm}$ & $\phi_{\mathrm{F}}$ & $\tau_{\mathrm{F}} / \mathrm{ns}$ & $\phi_{\mathrm{T}}$ & $\phi_{\Delta}$ & $\phi_{\mathrm{IC}}$ & $k_{\mathrm{F}}^{b} / \mathrm{ns}^{-1}$ & $k_{\mathrm{NR}}^{b} / \mathrm{ns}^{-1}$ & $k_{\mathrm{IC}}^{b} / \mathrm{ns}^{-1}$ & $k_{\mathrm{ISC}}^{b} / \mathrm{ns}^{-1}$ \\
\hline Polymer & 630 & - & 672 & 42 & 0.00037 & 0.050 & 0.006 & - & 0.9936 & 0.00740 & 19.99 & 19.87 & 0.12 \\
\hline Indigo & 610 & 22140 & 653 & 43 & 0.0023 & 0.140 & $0.0066^{a}$ & 0.0012 & 0.991 & 0.0164 & 7.12 & 7.12 & 0.0084 \\
\hline
\end{tabular}




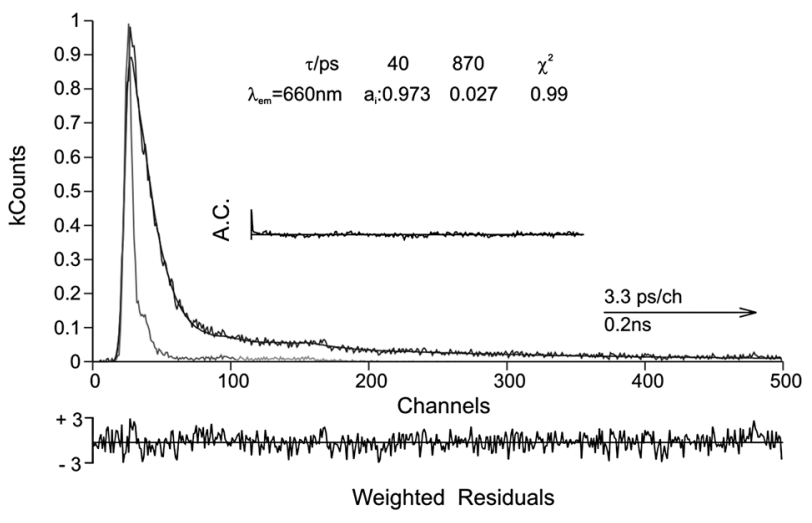

Fig. 3 Fluorescence decay and pulse instrumental response (short pulse) for the indigo polymer in DMF at $T=293 \mathrm{~K}$. Shown as insets are the decay times and pre-exponential factors. Also shown are the weighted residuals, autocorrelation functions (A.C.) and the $\chi^{2}$ values for a better judgment of the quality of the fits.

calculations $^{6}$ and by ultrafast real-time vibrational spectroscopy ${ }^{27,28}$ it was concluded that indogocarmine (the water soluble sulfonate indigo derivative) undergoes an intramolecular single-proton transfer in its ${ }^{1} \pi, \pi^{*}$ excited state.
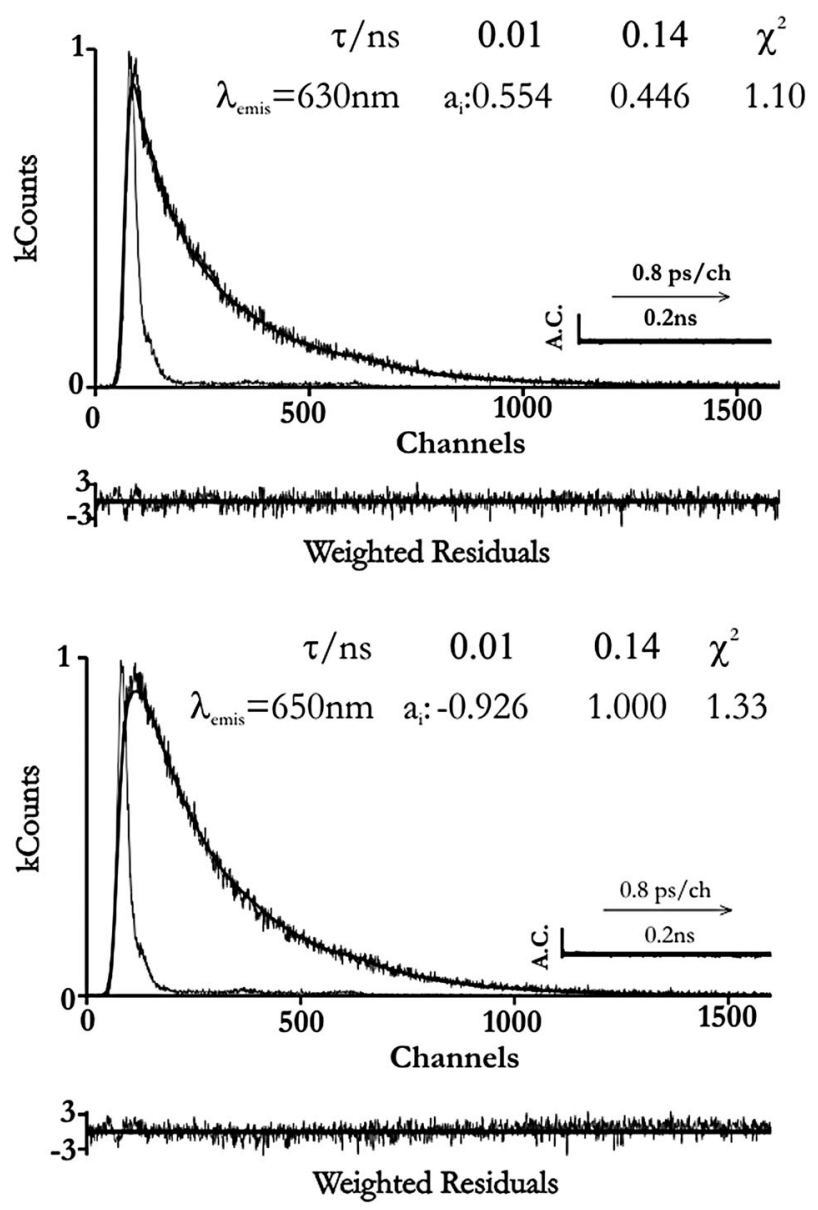

Fig. 4 Fluorescence decay and pulse instrumental response for indigo in DMF, collected at two different emission wavelengths at $T=293 \mathrm{~K}$. Shown as insets are the decay times and pre-exponential factors. Also shown are the weighted residuals, autocorrelation functions (A.C.) and the $\chi^{2}$ values for a better judgment of the quality of the fits.
Moreover, also worth noting is the short value for the fluorescence lifetime ( $\sim 40-50 \mathrm{ps}$ ) of the polymer, when compared with that of indigo itself, with values ranging between 130-140 ps (depending on the solvent; see Table 1, Fig. 4 and ref. 7, 16 and 25), suggesting that, as with other organic conjugated polymers, ${ }^{17,29}$ on-chain energy migration, along chromophoric units (of different segmental size), is responsible for the excited state deactivation of the polymer. Moreover, the absence of a rising component (which is present in indigo and associated with an instantaneously formed species which further transfers its proton in the excited state from the $\mathrm{N}-\mathrm{H}$ to the $\mathrm{C}=\mathrm{O}$ group) indicates that, in the polymer, either this process competes (inefficiently) with energy migration or is purely absent.

It is worth stating that the decay of the polymer is not single exponential. Indeed, an additional long-lived component is needed to properly fit the decays; however, this component is relatively insignificant since the associated pre-exponential factor represents less than $3 \%$ of the (concentration) species generated at time zero. The need (and presence) for this additional component is suggested to be related to some of the polydispersity associated with the polymer or, given the different chromophoric contributions to the structure of the polymer, to a partial emission of these two partially isolated units, indigo (keto structure) and tautomeric $\mathrm{N}$-acetylindigo.

\subsection{Determination of $\phi_{\text {ISC }}$ from photoacoustic experiments}

As with indigo, we were unable to observe any clear triplet-triplet transient signal; therefore, in order to characterize the triplet state of this polymer, we have performed, as with indigo itself, ${ }^{8}$ time resolved photoacoustic (PAC) experiments. In the case of systems with negligible volume changes, as it is the present case, time-resolved PAC measures directly the heat released in the decay of transients formed by pulsed laser excitation.

In a typical PAC experiment one obtains the amount of energy released through the radiationless channels, together with the lifetimes associated to these processes. ${ }^{8,20}$ In order to have these values for an unknown sample, the photoacoustic wave is first obtained-under identical conditions, including the same optical density at the excitation wavelength - for the solvent and for a reference compound, which decays totally through radiationless processes during a time shorter than the transducer resolution $(<10 \mathrm{~ns})$. From the deconvolution of the sample and reference signals, the fraction(s) of heat released in the formation of the sample transient(s) are obtained. Since it was previously established that more than $99.9 \%$ of the quanta loss in indigo are from radiationless processes, ${ }^{8}$ indigo itself was used as the photoacoustic reference for these experiments. In practical terms, the experimental procedure consists in measuring the reference, sample and solvent photoacoustic signal under the same conditions at different laser energies; the experiments are carried out in order to avoid or minimize bi-photonic effects. In the current experiment four laser energies were used to excite the polymer solution.

As can be observed in Fig. 5, where the waves of a typical PAC experiment are presented - solvent, reference (indigo in the present case) and the polymer - the PAC wave of the polymer did not show significant differences relative to the reference (indigo), either in the presence or absence of oxygen. Moreover, since no 


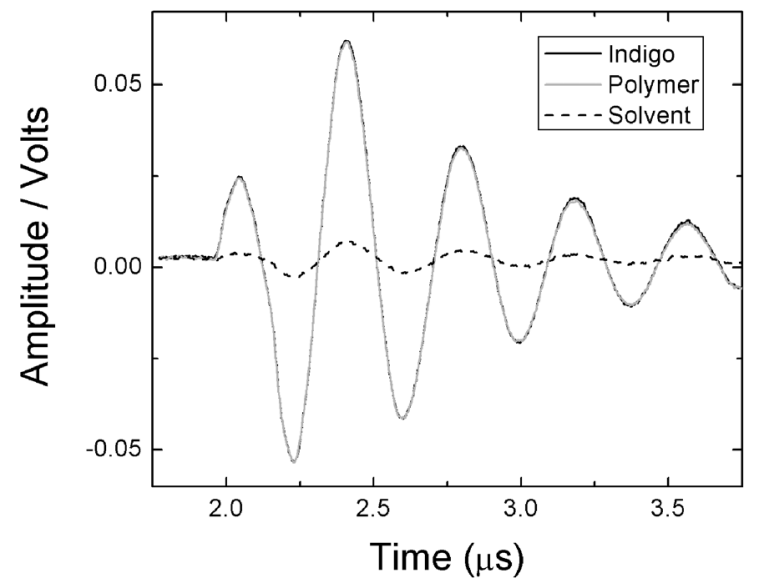

Fig. 5 Example of a typical PAC experiment with the waves for $100 \%$ laser intensity: reference wave (indigo, full black line), sample wave (full grey line); sample and reference in DMF (solvent) at $293 \mathrm{~K}$.

bi-photonic effects could be detected, there was no justification to obtain the energy released (and deposited as heat) by extrapolation to excitation energy values of zero (usual procedure used in our lab for these experiments), and the value was obtained as the mean value of four different experiments (see ESI $\dagger$ ) giving a quantum yield value - for the energy released in the form of heat—of $\phi_{1}=0.997 \pm 0.02$.

In the case of indigo (and the same occurs with the polymer, see Table 1), the triplet is formed very rapidly (within a few hundred ps for indigo and 40-50 ps for the polymer) thus contributing to a prompt heat released fraction $\left(\phi_{1}\right)$; moreover its decay in deaerated solutions is much slower $\left(\tau_{\mathrm{T}} \approx 30 \mu \mathrm{s}^{16}\right.$ in the case of indigo) than the time window of our experiment and is therefore not detected by the transducer used. The value of $\phi_{1}$ corresponds - in this system - to the energy fraction deposited in the $\mathrm{S}_{1}-\mathrm{S}_{n}$ decay and $\mathrm{T}_{n}-\mathrm{S}_{1}$ intersystem crossing. Thus, with the $\phi_{1}$ value measured by PAC, knowledge of the energies of the excited states involved $\left(\mathrm{S}_{1}\right.$ and $\left.\mathrm{T}_{1}\right)$, and the yield of fluorescence $\left(\phi_{\mathrm{F}}\right)$ it is possible to determine the quantum yields of the nonradiative processes. ${ }^{8}$

The energy balance for the formation of the triplet state requires that the energy of the laser pulse $\left(E_{h \nu}\right)$ minus the sum of the energies released as heat in the formation of the triplet $\left(\phi_{1} E_{h \nu}\right)$ and the energy lost radiatively $\left(\phi_{\mathrm{F}} E_{\nu_{\max }}\right)$ must equal the energy stored in the triplet state according to the following equation: ${ }^{8}$

$$
\phi_{\mathrm{T}} E_{\mathrm{T}}=\left(1-\phi_{1}\right) E_{h \nu}-\phi_{\mathrm{F}} E_{\nu_{\max }}
$$

where $E_{\nu_{\max }}$ is the energy of fluorescence (taken as the energy at the maximum fluorescence intensity considered as the Gaussian center value of the fluorescence band) and $E_{h \nu}$ (the energy of the laser pulse) equal to $2.33 \mathrm{eV}$. In the case of polymeric indigo, and assuming the value of the triplet energy of indigo, $134.7 \mathrm{kcal} \mathrm{mol}^{-1}(1.05 \mathrm{eV})$, and the values of $\phi_{\mathrm{F}}=0.00037$ (Table 1) together with the energy of the singlet state $\left(E_{\nu_{\max }}\right)$ $42.43 \mathrm{kcal} \mathrm{mol}^{-1}(1.84 \mathrm{eV})$ a value of $\phi_{\mathrm{T}}=0.0060$ was obtained; a value which is very similar to that found for indigo $(0.0066){ }^{8}$

Summarizing at this stage: the quantum yield of fluorescence decreases one order of magnitude for the polymer when compared to indigo, Table 1; this, together with the value for

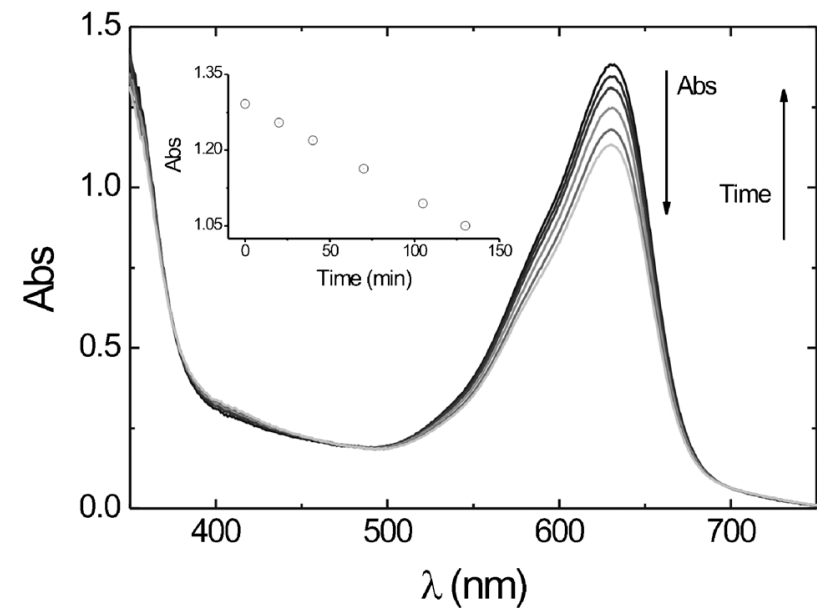

Fig. 6 Polymeric indigo in DMF, irradiated at $335 \mathrm{~nm}$ and $293 \mathrm{~K}$, followed by UV-Vis absorption; as the inset shows the plot of the variation in absorbance at $630 \mathrm{~nm}$, for the initial times, from which, compared with indigo under the same experimental conditions, the $\phi_{\mathrm{R}}$ value could be obtained.

the intersystem crossing yield ( 0.0066 for indigo vs. 0.0060 for the polymer), leads to an even higher $\phi_{\text {IC }}$ value for the polymer, thus showing that the process behind this decay route is more efficient in the polymer.

\subsection{Stability of polymeric indigo towards light}

Indigo and the polymeric indigo were irradiated with $\lambda_{\mathrm{exc}}=$ $335 \mathrm{~nm}$ at different time intervals and the UV/Vis absorption spectra were obtained, Fig. 6. From the plots shown in Fig. 6 and eqn (2) and (3) (Experimental section), the $\phi_{\mathrm{R}}$ was obtained for indigo in DMF (0.0078) - which compares well to the previously reported value ${ }^{10}$ - and for polymeric indigo $(0.0030)$, respectively. This value is significantly lower than that of indigo ( $\sim 2.7$ times $)$ which indicates that the indigo polymer has additional stabilization routes to (photo)degradation towards light. It is worth noting that although not investigated for the current polymer, the major product resulting from the photodegradation of indigo was found to be isatin. ${ }^{10}$

\section{Conclusions}

The photophysics and photochemistry of a polymeric indigo have been investigated. It was found that the polymer presents similar spectral and photophysical properties when compared to indigo itself. However, its fluorescence quantum yield is one order of magnitude lower than those presented by indigo. The efficient internal conversion radiationless process is found to occur both in the polymer and indigo. Nevertheless, and in contrast with indigo, where intra- or inter-molecular proton transfer ${ }^{6,7,25-28,30}$ (between the $\mathrm{N}-\mathrm{H}$ and $\mathrm{C}=\mathrm{O}$ groups) was attributed to the efficiency of this specific channel, in the polymer a plausible explanation (not necessarily unique) consists in an energy migration process between different indigo chromophoric units. This is related to the lower value of the (photochemistry) reaction quantum yield for the polymer which indicates that this has additional stabilization routes to (photo)degradation towards light. 


\section{Acknowledgements}

We are grateful to POCI (project PTDC/QUI-QUI/099388/ 2008), Fundação para a Ciência e a Tecnologia (FCT) and FEDER for further funding. R.R. acknowledges FCT for a PhD grant (SFRH/BD/38882/2007).

\section{References}

$1 \mathrm{~J}$. Balfour-Paul, in XXth International Congress of History of Science, Liége, Belgium, 1997.

2 J. Balfour-Paul, Indigo in the Arab World, RoutledgeCurzon, London, 1997.

3 J. Balfour-Paul, Indigo, British Museum Press, 2000.

4 L. Meijer, N. Guyard, L. A. Skaltsounis and G. Eisenbrand, Indirubin, the red shade of indigo, Life in Progress Editions, Roscoff, France, 2006.

5 L. G. Wang and S. K. Mencher, in Indirubin, the red shade of indigo, ed. L. Meijer, N. Guyard, L. A. Skaltsounis and G. Eisenbrand, Life in Progress Editions, Roscoff, France, 2006, pp. 247-258.

6 S. Yamazaki, A. L. Sobolewski and W. Domcke, Phys. Chem. Chem. Phys., 2011, 13, 1618-1628.

7 J. Seixas de Melo, R. Rondão, H. D. Burrows, M. J. Melo, S. Navaratnam, R. Edge and G. Voss, J. Phys. Chem. A, 2006, 110, 13653-13661.

8 J. S. Seixas de Melo, C. Serpa, H. D. Burrows and L. G. Arnaut, Angew. Chem., Int. Ed., 2007, 46, 2094-2096.

9 M. Montalti, A. Credi, L. Prodi and M. T. Gandolfi, Handbook of Photochemistry, CRC Press, Boca Raton, FL, 2006.

10 M. M. Sousa, C. Miguel, I. Rodrigues, A. J. Parola, F. Pina, J. S. Seixas de Melo and M. J. Melo, Photochem. Photobiol. Sci., 2008, 7, 1353-1359.

11 G. Heller, Zeitschr. Farben- und Textilchem., 1903, 2, 329-332.

12 G. Voss, M. Drechsler, S. Eller, M. Gradzielski, D. Gunzelmann, S. Mondal, S. van Smaalen and C. S. Voertler, Helv. Chim. Acta, 2009, 92, 2675-2697.
13 H. Tanaka, K. Tokuyama, T. Sato and T. Ota, Chem. Lett., 1990, $1813-1816$.

14 J. G. Mei, K. R. Graham, R. Stalder and J. R. Reynolds, Org. Lett., 2010, 12, 660-663.

15 R. Stalder, J. G. Mei and J. R. Reynolds, Macromolecules, 2010, 43, 8348-8352.

16 J. Seixas de Melo, A. P. Moura and M. J. Melo, J. Phys. Chem. A, 2004, 108, 6975-6981.

17 J. Pina, J. Seixas de Melo, H. D. Burrows, A. L. Maçanita, F. Galbrecht, T. Bunnagel and U. Scherf, Macromolecules, 2009 , 42, 1710-1719.

18 G. Striker, V. Subramaniam, C. A. M. Seidel and A. Volkmer, J. Phys. Chem. B, 1999, 103, 8612-8617.

19 L. G. Arnaut, R. A. Caldwell, J. E. Elbert and L. A. Melton, Rev. Sci. Instrum., 1992, 63, 5381-5389.

20 F. A. Schaberle, R. M. D. Nunes, M. Barroso, C. Serpa and L. G. Arnaut, Photochem. Photobiol. Sci., 2010, 9, 812-822.

21 F. Pina, L. Moggi, M. F. Manfrin, V. Balzani, M. W. Hosseini and J. M. Lehn, Gazz. Chim. Ital., 1989, 119, 65-67.

22 S. T. Hoffmann, H. Bassler and A. Kohler, J. Phys. Chem. B, 2010, 114, 17037-17048.

23 J. Seixas de Melo, H. D. Burrows, M. Svensson, M. R. Andersson and A. P. Monkman, J. Chem. Phys., 2003, 118, 1550-1556.

24 R. Kersting, U. Lemmer, R. F. Mahrt, K. Leo, H. Kurz, H. Bassler and E. O. Gobel, Phys. Rev. Lett., 1993, 70, 3820-3823.

25 J. S. Seixas de Melo, R. Rondão, H. D. Burrows, M. J. Melo, S. Navaratnam, R. Edge and G. Voss, ChemPhysChem, 2006, 7, 2303-2311.

26 Y. Nagasawa, R. Taguri, H. Matsuda, M. Murakami, M. Ohama, T. Okada and H. Miyasaka, Phys. Chem. Chem. Phys., 2004, 6, 5370-5378.

27 I. Iwakura, A. Yabushita and T. Kobayashi, Bull. Chem. Soc. Jpn., 2011, 84, 164-171.

28 I. Iwakura, Phys. Chem. Chem. Phys., 2011, 13, 5546-5555.

29 J. Pina, H. D. Burrows and J. S. Seixas de Melo, in Specialist Periodic Reports in Photochemistry, ed. A. Albini, RSC, Cambridge, 2011, pp. 30-64.

30 I. Iwakura, A. Yabushita and T. Kobayashi, Chem. Lett., 2009, $1020-1021$. 